\title{
An Overview on Multiple Unmanned Aerial Vehicle Control through Single Controller
}

\author{
Ujjwal Sharma \\ MRIIRS \\ Faridabad, Haryana
}

121004

\author{
Vivek Rawal \\ MRIIRS \\ Faridabad, Haryana \\ 121004
}

\author{
Veena Tayal \\ MRIIRS \\ Faridabad, Haryana \\ 121004
}

\begin{abstract}
Nowadays, the demand for a reliable autonomous application is increasing enormously. A fully autonomous body could reduce human involvement and increase efficiency of the desired function. Managing a single task is quite easy but performing same task over multiple times with same accuracy requires big effort. Therefore designing a system which could match the complexity of same work repeatedly will bring a new revolution. In this paper, we are developing a system through which multiple aerial drones can be controlled through single controller. We are focusing on UAVs and in concern to achieve this we aim at managing minimum distance at low level and at high level, they should not disturb the path of other flying drone. They should re-center their location through sensor of collision and GPS coordination. This research will bring many applications in control, for example rescue missions and in agriculture.
\end{abstract}

\section{Keywords}

UAV (Unmanned Aerial Vehicle), Drone, path trajectory.

\section{INTRODUCTION}

The beginning of 21st century, the era of technology has brought many changes in the field of autonomous industry and UAVs are regularly positioning its popularity in the market with its design and range of sizes. The most common type of UAV are drones. UAVs are of multiple types based on its flying firmware [1], Multi Rotor Drones, Fixed Wing Drones, Single Rotor Helicopter and Fixed wing Hybrid VTOL. Generally single pilot hold single drones at a time and regularly get updated with its geographical location at the ground level. Drones are not bound to only single application or use, they came with maximum flexibility. We just need to develop a system with added features to get multiple result using single input. When we define a similar task and ask to perform through multiple UAVs, it requires a change in its firmware which can be modified through scaling brains of the drones. This will reduce the time for achieving an objective with maximum efficiency.

Regular improvement in design of drones and its autopilot brings various real life problems more scalable and easy to handle. Drones and UAVs all came with embedded sensors and predefine control system. They helps in flight of aerial vehicle with GUI and manual control. Added features of drones such as sensors in autopilots for hobbyist UAVs are described by Chao et al [2]. All developed built-in sensors brought growth in firmware through open source and increases capabilities of the UAVs. These sensors provide autopilot handling to next level. Various controller are already made for handling drones such as Sparky system [3] and Pixhawk controller [4] which is built over ArduCopter firmware through open source that helps to enable control over multiple aerial drones using single controller.
ArduCopter firmware can be used to maintain minimum distance maintenance between two flying copters. We can also add the features of low level to high level which will leverage the design and behavior for multiple copters.

Research and studies have shown large benefits of implementing such application; it will not only reduce cost but also saves time. Single pilot can control many number of drones at once which will eventually increase efficiency of the task. When it comes to static and dynamic behavior of the UAVs, the stability increases with same holding agreement and enhancement in the positioning due to spatial dispense holding agreement in space and height. Different types of UAVs have its own advancement in terms of flight, firmware and degree of automation as rotary wing aerial vehicles provide better result for spy and examination than conventional fixed wing aircraft as flight and drift can be possible in a finite area but its control is quite difficult because of dynamic uncertainty and more sensitive to disorder. There is a need to know the number of UAVs that can be control by single controller at a time. As the maximum number of sensors, large amount of data and executable command in multiple UAVs will lead to excessive growth of cognitive application. Dynamic implementation of controls and human-system interaction criticality will affect system conduct. Control of UAVs depend upon multiple factors such as managerial control and range of manual control. In paper [18] M.L. Cummings discuss about the number of aerial vehicle, a controller could hold and the amount of wait time for human- system communication and its effect on flight.

Single control over multiple UAVs allows achieving faster rescue as more number of copters are more scalable than single one. It only requires hardware as Arduino Uno and transceivers. These are also helpful in close observation and careful examination. Various approaches has been discussed mathematically in response to path outlining [5] among which the most known one is Rapidly-exploring Random Trees (RRT) [6, 7] and $\mathrm{A}^{*}$ and $\mathrm{D}^{*}[17]$. When it comes to path outlining pseudo spectral method has been early enforce to resolve such problem [8]. Different kind of approaches been discussed in many paper, multi-objective evolutionary algorithms [9], particle swarm optimization [10, 11] and evolutionary techniques [12, 13]. In paper [5] the author presented an approach to solve clash apprehension and verdict. One of the best approach to solve clash apprehension is speed position [14] which state speed position for all entire aerial vehicle that participate in encounter can be calculated centralize. Another author in paper [15] define an approach based on mixed-integer linear programming (MILP) which alter the speed of considerable flying copter even could not succeed in solving all conflict. However other approaches solves couple conflict [16] but fail to consider all flying copters. 
When we talk about regular development, low level behaviors are more developed than high level which is keen point of interest for future research. The paper are describe in following sections, section 2 deals with previous research in this area, section 3 describes the approach and methods used, section 4 describe the result and section 5 discuss futuristic application of this work and section 6 provides references.

\section{PREVIOUS WORK}

There are number of research works have been done on flying UAVs.Burke et al. formally introduce a platform for the flight of swarms of drones [19]. Multiple flying copters in a defined formation have been achieved at Georgia Tech [20]. Research on this has been done using different factors such as predefined flight paths but when it comes to full control over drones at a time it requires more study and work. There are many real life application need to be handle with full control over autonomous flight drones. However to achieve this situation, it requires some complexity to be solve to enable complex formation and behaviors so we follow simple building blocks [21] mechanism based on Braitenburg's novel vehicle. This will help us in maintaining distance between two flying copters.

Developing these functionality in each copter will lead us to keep safe distance to flight multiple copters remotely. Based on fixed path planning problem, Dantzig et al., proposed an ineger linear program [22]. Many algorithm are designed for path planning, among which Tabu Search (Ts) heuristic algorithm [23, 24] was the early approach which deals path travel without trapped in local path. Along with flight movement we must ensure the correct formation of flight in response to relative motion of different UAV and their longitudinal as well as sideways fuzzy curb. Their relative locations are entitled to perform required command and that enforces other nearby flying UAV to fly without collision with firmness of controller. Fuzzy control of the UAV could be consider using Q-Learning approach which is part of reinforcement artificial intelligence. There is a need for corresponding control, autonomous bond control and formation control along the site of remote and local centralize control In paper [25] Singh proposed the non-definite flexible formation using two different UAV who guides other to follow and establish the close-loop cohesion of the controller. In another paper [26] Giulietti design the LQ-Servo that helps to manage the formation with whirlwind interruption. There are many more work conducted in West Virginia University which drove three UAV to form a formation by precarious assessment and in Beihang University an optimistic control system is designed by Lingpei Zong on different multiple system[27]. On the other side Zheng develop a disburse linear feedback control in verge to manage the formation [28]. The flexibility of copters has been increased along with flight at fast speed in littered surrounding [29], balancing copter [30], self-controlling platform and field calculating [31],corresponded apprehend of ground vehicle [32], etc. All of these follows GPS coordinates for flight movement. There are some authors [33, 34] who make flight using motion capture system within a building.

Our first priority is to make an autonomous flying copter by using all possible sensors. GPS technology is used to flight UAVs without human interaction using inertial sensor [18]. Where GPS technology fails to work then we can use dead calculation for position but it is an older technology nowadays. When we talk about maintaining height, some authors have uses ultra sound sensors in their research. Their research were successful in achieving autonomous flight but they fail to control over direction. They tried number of times to get success using monocular camera as sensors [36] and function optical surveying by using Moire arrangement that helps in controlling six degree of ability to flight autonomous. This arrangement is also used in paper [18] that describe perception dependent stability and productivity in tracking discipline for a helicopter. Paper shows uses of two cameras for this in which one is located at the ground level and another is located onboard in such a way both cameras faces to each other. When we talk about large area or dynamic area which could not be stimulated at the run time for a smooth drive so in that case, one reference point is needed at which UAV could move or decide its flight. Ground camera can be consider as referential frame for flight movement and onboard camera will follow reference and decide its flight trajectory. Growth in the field of automation have been consider along three loops, human legislative jurisdiction and ranked jurisdiction over single and multiple UAVs [18].There are more few authors $[38,4]$ who make flight but all were indoor and under established design but lead to EKF-based Vision SLAM structure that build a low-verdict three dimension plan. There is a need for external visual firmware that can be adopt for flight inertial sensor knowledge.

Designing best solution for clash-free path for multiple flying UAV are advantages to develop with multiple local minimum in maximum scenario. Therefore local expansion approach such as gradient based approach are not helpful in solving conflict. However the global approach of GA or Particle swarm optimization (PSO) which has low level of calculation overhead and faster problem approach and could be a better option for this and for path defining NP-Hard [39, 40] is optimal solution due to its dynamic changes in trajectory.

\section{METHODS}

The aim for flying multiple UAVs using single controller can be achieve at low level by considering distance maintenance between multiple UAVs. Each UAV makes fly using different parameters, which help them to sense nearby available UAV but this can be implement more precisely using two simple principles. When two copters are flying at a far distance from each other, they must come closer automatically whereas when they are flying at a nearby distance in which chances of collision may occur, they must move apart from each other. With this principle of distance maintenance, we can reduce more complex behavior of drone flight. We can use received signal strength indicator (RSSI) to deal with such situation but as it uses radio frequency, it may get distortion sometime. Sonar is another better solution for identifying nearby copters. It is also stated that sonar is not an efficient option for this as it works better under the range of 5 to 11 meters [2]. Another technique that we have as an option is computer vision through which nearby copters can be recognize by vision camera that will be on the copter itself. There is also an issue regarding this as we need a 360 camera vision which is quite difficult to capture using copters. GPS navigation technique is quite reliable in this situation but one issue is also available with this that GPS navigation is not always with precise accuracy. The GPS navigation can be added with modified hardware that will record data of its flight and send to all nearby copter. We could consider different scenario for describing its implementation such as assume complete system and try to resolve non-cramped controls. In second scenario we break the whole control into simpler movements and then collaborate the entire assumption to define complete path trajectory. This approach could be easily used for the flight of quadrotar UAV that switch its control repeatedly 
from left to right and vice versa. This problem can be resolved by taking all its three dimension constant and their flight angle at zero degree. Among three dimension only altitude changes randomly and other parameter are kept constant but when it is also zero or constant then stability will be achieve. Similarly this low level behavior can be inherit for high level behavior.

\subsection{UAVs Communication}

For a successful flight of multiple UAVs, it is much important to be aware about time interval between sensor for direction and the receiver response to such command as shown in Fig 1. In the paper [18] M.L Cummings et al. describe about utilization value for human-automation interaction and wait time for flying copter communication. Transceiver attached to copters needs full information through GPS about longitude, latitude, altitude and compass orientation. The longitude value could be obtained from Arduino Uno that will display on the screen. GPS HDOP is the best option to get coordinates with high accuracy. Compass orientation lies in the range of 0-360 degrees where 0 degree points towards north. It helps in aligning the copter in the ideal direction. Multiple copter will follow a single copter. The data is valid only if GPS HDOP is within range of 1.5 meters where as if it is higher than this then data is stored and current data is communicate to other nearby flying drones for coordinates. This will help in maintaining distance when the distance will be close between copter or distance is far then flying speed of the copter will glitch and appropriate distance will be maintained. We are at verge of drawing an autonomous UAV that could be managed by onboard controller only without the help of predefined trajectory.

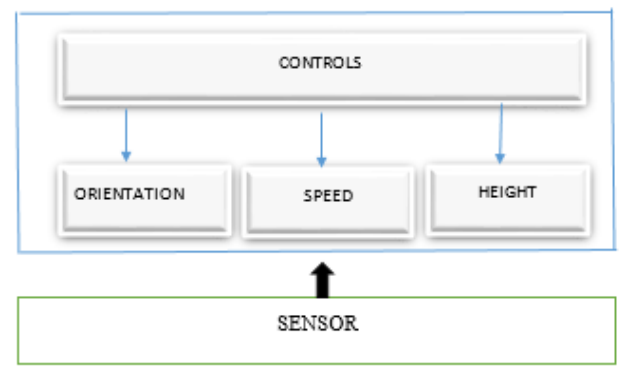

Fig 1: UAVs communication

\subsection{Clash-Free Trajectory}

Designing a smart system that can define clash free trajectory in dynamic surrounding need scalable firmware. System should auto sense for collision in prior to contact. The system need to be loaded with full information and data about initial dimension for trajectory, framework for each nearby flying model of UAV and their geographical location of flying. A simple solution to avoid clashes would be collecting trajectories of all flying UAV in a deterministic sample rate and minimize the all possible clash sample. Define a path matching algorithm in predefined approach that met with dynamic environment in real time to gain absolute speed and relative localize data. Another objective to overcome this problem is regional mapping which let UAV to flight in deterministic path. Clash free trajectory also include aligning of multiple UAV which could be resolve by EKF data fusion and outlining trajectory through covariance validation. Data obtained by previous flight trajectory will result for future path planning but it will be too complex to implement so we have to use simple equation for low level behavior and high level behavior such as back stepping controller and distributive control with known and unknown localization of parameter. Flight of UAV must be reference with the flying environment.

\subsection{Degree of Automation}

It is highly difficult to supervise each flying UAVs at the same time. Degree of automation or level of automation will decide the amount of autonomy and amount of labor that an autonomous UAVs can reduce and to which extent. The UAVs having higher level of autonomy reduces the human attention and increase the neglect time. When it comes to decision making behavior of the system it can be fully autonomous or could be handover manually even at small decision of data refining [23]. When it come for sensing and prediction calculation there are Kalman Filters for refining correct value in a noisy environment. Redefining of SLAM algorithm from two dimension environment to three dimension environment. When it comes to system reliability where the approach for decision making require less flexibility and chances for system turndown is very less, then it is often the optimal solution in terms of overhead [24]. However, human negligence about environment could also affect the performance of UAVs [41]. During flight of UAVs single error could cause more damage whereas the system must adapt the control and degree of automation for excellence performance. Designing UAV with laser that helps in defining range in anonymous and destructive environment, whether it is inside building or outside. Different sensor are require for flight simulation.

Table 1: Flight condition simulation with sensor

\begin{tabular}{|l|l|}
\hline Flight Condition & Sensor \\
\hline Acrobatic & Gyroscope \\
\hline Balanced & Gyroscope, Accelerometer \\
\hline High & $\begin{array}{l}\text { Gyroscope, Accelerometer, } \\
\text { Barometer }\end{array}$ \\
\hline Direction & $\begin{array}{l}\text { Gyroscope, Accelerometer, } \\
\text { Compass }\end{array}$ \\
\hline Location & GPS \\
\hline
\end{tabular}

\section{RESULT}

\subsection{Communication Time}

Communication time between sensor and receiver will be generally low as transmitter uses GPS coordinates for its flight. Communication time could be observe as a mean after flight of UAVs multiple times and record the data. In paper [18] communication time refer as interaction time when interacted with UAV produces same level of wait time with some divergence based on level of automation of UAV.

\subsection{Response time between two UAVs}

Whenever transmitter transmits the signal for all UAVs. The response time that can be observe in UAV could vary depending on the flight orientation and wind speed. Response will be collected in the form of queue. There may be unusual record be recorded during its flight and it require high level of research.

\subsection{Flight alignment of UAVs}

Single UAV not overhead for flight orientation but when it comes to fly multiple UAVs in a considerable outline then it requires high knowledge about its orientation, level of automation, distance between nearby UAVs and wind direction. Flying UAVs against the wind requires high automation and adaptability behavior. Multiple scenario with 
multiple parametric factors need to be examine such as keeping one UAVs as stable and flying second UAV around it to record its dynamic response. Still our first priority is to maintain a static distance between consecutive flying UAVs. High level behavior need more research for a successful flight.

\subsection{Single Controller}

Already developed controller such as Pixhawk and Sparky system are available but the requirement for lighter controller is still in need. Through these controller multiple UAVs could fly and be able to control but when it comes to more complicated route, these controller has the limitation. Complexities are more but need to be resolve.

\section{DISCUSSION}

The modified firmware of the UAVs and receiver will help to maintain an appropriate distance among them. Efficiency of the work will increase and multiple UAVs can be control by single pilot in real time. The main application of using such application is in agriculture where large area can be cultivated or harvested by single controller. Another important application is rescue and search where drones can help in searching the victim and task can be performed by single pilot. There are more area to be consider for future work while these technologies i.e. GPS, Sonar are not reliable with 100 percent accuracy. The waiting time and level of flight of UAV depends on the level of automation.

\section{REFERENCES}

[1] Types of Drones - Explore the Different Types of UAV's. (2017, February 03). Retrieved from http://www.circuitstoday.com/types-of-drones

[2]. Chao, HaiYang, YongCan Cao, and YangQuan Chen. Autopilots for Small Unmanned Aerial Vehicles: A Survey. International Journal of Control, Automation and Systems 8, no. 1 (February 17, 2010): 3644.

[3] SPARKY 2.0. (n.d.). Retrieved from https://www.dronematters.com/power-system/flightcontrollers/sparky-2-0.html

[4]Pixhawk Autopilot - PX4 Autopilot Project, https://pixhawk.org/modules/pixhawk

[5] J. K. Kuchar and L. C. Yang, "A review of conflict detection and resolution modeling methods," IEEE Transactions on Intelligent Transportation Systems, vol. 1, pp. 179-189, 2000.

[6] S. M. Lavalle, J. J. Kuffner, and Jr., "Rapidly-Exploring Random Trees: Progress and Prospects," in Algorithmic and Computational Robotics: New Directions, 2000, pp. 293-308.

[7] Y. Lin and S. Saripalli, "Path planning using 3d dubins curve for unmanned aerial vehicles," in Proceedings of the International Conference on Unmanned Aircraft Systems (ICUAS2014), Orlando, USA, 27-30 May 2014, pp. 296-304.

[8] S. Vera, J. A. Cobano, G. Heredia, and A. Ollero, "An hpadaptative pseudospectral method for collision avoidance with multiple uavs in real-time applications," in IEEE Int. Conf. Robotics and Automation (ICRA), Hong Kong, China, May 31 - June 72014.

[9] A. J. Pohl and G. B. Lamont, "Multi-objective UAV mission planning using evolutionary computation," in Winter Simulation Conference, 2008, pp. 1268-1279.
[Online]. Available: http://dx.doi.org/10.1109/WSC.2008.4736199

[10] D. Alejo, J. A. Cobano, G. Heredia, and A. Ollero, "Particle Swarm Optimization for collision-free 4d trajectory planning in unmanned aerial vehicles," in Proceedings of the International Conference on Unmanned Aircraft Systems (ICUAS2013), Atlanta, USA, 28-31 May 2013, pp. 298-307.

[11] M. Pontani and B. A. Conway, "Particle Swarm Optimization Applied to Space Trajectories," Journal of Guidance Control and Dynamics, vol. 33, pp. 14291441,2010

[12] J. A. Cobano, R. Conde, D. Alejo, and A. Ollero, "Path planning based on genetic algorithms and the montecarlo method to avoid aerial vehicle collisions under uncertainties," in Proc. IEEE Int Robotics and Automation (ICRA) Conf, 2011, pp. 4429-4434.

[13] R. Vivona, D. Karr, and D.Roscoe, "Pattern-based genetic algorithm for airborne conflict resolution," in AIAA Guidance, Navigation and Control Conference and Exhibit, Keystone, Colorado, August 2006.

[14] J. A. Cobano, D. Alejo, A. Ollero, and A. Viguria, "Efficient conflict resolution method in air traffic management based on the speed assignment," in Proceedings of the 2nd International Conference on Application and Theory of Automation in Command and Control Systems, ser. ATACCS '12. Toulouse, France, France: IRIT Press, 2012, pp. 54-61. [Online]. Available:

http://dl.acm.org/citation.cfm?id=2325676.2325684

[15] A. Vela, S. Solak, W. Singhose, and J.-P. Clarke, "A mixed integer program for flight-level assignment and speed control for conflict resolution," in Decision and Control, 2009 held jointly with the 2009 28th Chinese Control Conference. CDC/CCC 2009. Proceedings of the 48th IEEE Conference on, dec. 2009, pp. 5219 -5226.

[16] H. Erzberger, "Automated conflict resolution for air traffic control," in Proceeding International Congress Aeronautical Sciences, 2006, pp. 179-189.

[17] A. Stentz and I. C. Mellon, "Optimal and Efficient Path Planning for Unknown and Dynamic Environments," International Journal of Robotics and Automation, vol. 10, pp. 89-100, 1993.

[18] Cummings, M. L., \& Mitchell, P. J. (2008). Predicting controller capacity in supervisory control of multiple UAVs. IEEE Transactions on Systems, Man, and Cybernetics-Part A: Systems and Humans, 38(2), 451460.

[19] Burkle, Axel, Florian Segor, and Matthias Kollmann. Towards Autonomous Micro UAV Swarms. Journal of Intelligent \& Robotic Systems 61, no. 14 (October 27, 2010): 33953.

[20]GT — News Center?:: Multiple UAVs Perform Autonomous Formation Flight, http://www.news.gatech.edu/2014/08/07/multipleuavsperform-autonomous-formation-flight

[21] Braitenberg, Valentino. Vehicles: Experiments in Synthetic Psychology. MIT Press, 1986.

[22] Systems (ICUAS), Denver, CO, USA, 9-12 June 2015. 
[23] Y. Lin and S. Saripalli, "Path planning using 3d dubins curve for unmanned aerial vehicles," in Proceedings of the International Conference on Unmanned Aircraft Systems (ICUAS2014), Orlando, USA, 27-30 May 2014, pp. 296-304.

[24] S. Vera, J. A. Cobano, G. Heredia, and A. Ollero, "An hpadaptative pseudospectral method for collision avoidance with multiple uavs in real-time applications," in IEEE Int. Conf. Robotics and Automation (ICRA), Hong Kong, China, May 31 - June 72014.

[25] Sahjendran. Singh, Phil Chandler, Corey Schumacher, Siva Banda, Meir Pachter. Nonlinear Adaptive Close Formation Control of Unmanned Aerial Vehicles. Dynamics and Control, vol10,2000,179-194.

[26] Fabrizio Giulietti, Mario Innocenti, Marcello Napolitano, Lorenzo Pollini. Dynamic and control issues of formation flight. Aerospace Science and Technology. vol 9,2005:65-71

[27] Zong lingpei $\square$ Xie Fan $\square$ Qin Shiyin. UAV Intelligent Optimal Control of Formation Flight Based on MAS. Chinese Journal of Aeronautics(Chinese). 29(5):13261333

[28] He Zhen, Lu Yuping. Decentralized Design Method of UAV Formation Flight Shape Keeping Controller. Journal of Aeronautics(Chinese). 29(Appendix Edition):S55-S60

[29] S. Scherer, S. Singh, L. Chamberlain, and S. Saripalli. Flying Fast and Low Among Obstacles. In Proc. ICRA, pages 2023-2029, 2007.

[30] A. Coates, P. Abbeel, and A.Y. Ng. Learning for control from multiple demonstrations. In Proc. ICML, pages, 144-151. ACM, 2008.
[31] T. Templeton, D.H. Shim, C. Geyer, and S.S. Sastry. Autonomous Vision-based Landing and Terrain Mapping

Using an MPC-controlled Unmanned Rotorcraft. In Proc. ICRA, pages 1349-1356, 2007.

[32] J.P. How, B. Bethke, A. Frank, D. Dale, and J. Vian. Real-time indoor autonomous vehicle test environment Control Systems Magazine, IEEE, 28(2):51-64, 2008

[33] G.M. Hoffmann, H. Huang, S.L. Waslander, and C.J. Tomlin. Quadrotor helicopter flight dynamics and control: Theory and experiment. In Proc. of GNC, Hilton Head, SC, August 2007.

[36] G.P. Tournier, M. Valenti, J.P. How, and E. Feron. Estimation and control of a quadrotor vehicle using monocular vision and moir`e patterns. In Proc. of AIAA GNC, Keystone, Colorado, 2006.

[38].In Ukraine, Tomorrows Drone War Is Alive Today. Defense

One, http://www.defenseone.com/technology/2015/03/ukraine tomorrows- drone-war-alive-today/107085/

[39] J. F. Gilmore, "Autonomous vehicle planning analysis methodology," in AIAAA Guidance Navigation Control Conference, 1991, pp. 2000-4370.

[40] R. J. Szczerba, "Threat netting for real-time, intelligent route planners," in IEEE Symp. Inf., Decis. Control, 1999, pp. 377-382.

[41] J. A. Cobano, R. Conde, D. Alejo, and A. Ollero, "Path planning based on genetic algorithms and the montecarlo method to avoid aerial vehicle collisions under uncertainties," in Proc. IEEE Int Robotics and Automation (ICRA) Conf, 2011, pp. 4429-4434. 